\title{
Image decomposition using a second-order variational model and wavelet shrinkage
}

\author{
Minh Phuong Tran* and Thanh Nhan Nguyen ${ }^{+}$ \\ * Applied Analysis Research Group, Faculty of Mathematics and Statistics, Ton Duc Thang University, Vietnam \\ ${ }^{+}$Department of Mathematics, Ho Chi Minh city University of Education, Vietnam
}

Received 29th August 2017; Accepted 16th June 2019

\begin{abstract}
The paper is devoted to the new model for image decomposition, that splits an image $f$ into three components $u+v+\omega$, where $u$ a piecewise-smooth or the "cartoon" component, $v$ a texture component and $\omega$ the noise part in variational approach. This decomposition model in fact incorporates the advantages of two preceding models: the second-order total variation minimization of Rudin-Osher-Fatemi (ROF2), and wavelet shrinkage for oscillatory functions.

This decomposition model is presented as an extension of the three components decomposition algorithm of Aujol et al. in [Dual norms and image decomposition models, Int. J. Comput. Vis. 63(1)(2005) 85-104]. It also continues the idea introduced previously by authors in [Denoising 3D medical images using a second order variational model and wavelet shrinkage, Imag. Anal. and Rec., Lecture Notes in Computer Science, 7325(2012), 138-145], for two components decomposition model. The ROF2 model was first proposed by Bergounioux et al. in [A second-order model for image denoising, Set-Valued Anal. and Var. Anal., 18(2010), 277-306], it is an improved regularization method to overcome the undesirable staircasing effect. The wavelet shrinkage is well combined to separate the oscillating part due to texture from that due to noise. Experimental results validate the proposed algorithm and demonstrate that the image decomposition model presents effective and comparable performance to other previous models.
\end{abstract}

Key Words: Image decomposition model, second-order total variation, ROF2 model, wavelet shrinkage.

Correspondence to: < tranminhphuong@tdtu.edu.vn >

Recommended for acceptance by Angel D. Sappa

https://doi.org/10.5565/rev/elcvia. 1162

ELCVIA ISSN:1577-5097

Published by Computer Vision Center / Universitat Autònoma de Barcelona, Barcelona, Spain 


\section{Introduction}

The problem of decomposing an image into several meaningful components has sparked considerable amount of research. Many decomposition models have been proposed so far in [10], [12], [13] aimed at splitting an image into two main components, one being the piecewise-smooth (also called "cartoon" part) that contains the geometrical information, and another one containing the oscillating components that result from both textural features and noise.

From a given image $f$ that has been corrupted by noise, or blurring effects, one extracts the cartoon part $u$ and the oscillating part $v$. The problem was first proposed for denoising purpose:

$$
\inf _{u \in B V(\Omega)} \frac{1}{2}\|f-u\|_{L^{2}(\Omega)}^{2}+\lambda T V(u, \Omega)
$$

where $\lambda \geq 0$ is a regularization parameter and $\Omega \subset \mathbb{R}^{n}(n \geq 2), T V(u, \Omega)$ represents total variation of $u$, assuming that $u \in B V(\Omega)$. It can refer to [7] for details of this functional space $B V$. Notice that the minimization problem has a unique solution since the functional energy is lower semi-continous and strictly convex.

In addition, the Meyer's model in [14] also follows to split an image $f$ as a sum $u+v$ between a cartoon $u$ (geometrical component) and a term $v$ takes care of textured components plus some additive noise. Then, the image decomposition is considered by minimizing a convex functional which depends on two variables $u$ and $v$ :

$$
\inf _{(u, v) \in X \times \mu B_{G}} \frac{1}{2 \lambda}\|f-u-v\|_{X}^{2}+J(u)
$$

where $X$ is the discrete functional space; $J(u)$ is the discretization of $T V(u, \Omega)$. One also denotes:

$$
B_{G(\mu)}=\mu B_{G}=\left\{v \in G \mid\|v\|_{G} \leq \mu\right\} .
$$

The model (2) is called the $B V-G$ algorithm, well implemented by J.F. Aujol et al. in [10], and Meyer Y. et al. in [2].

Nevertheless, the ROF model also has an important drawback, it favours piecewise constant solutions that occur most severely in image reconstructions, so-called staircasing effects. Thus, ameliorating this effect should be considered a priority in image decomposition problems. One way to overcome the staircasing in reconstruction is to introduce higher order derivatives into the energy. It has been studied extensively in many literatures. The higher-order variation regularization was first proposed in [6] and [20], and then the Total Generalized Variation (TGV) was also introduced in [4]. By extending the definition of the classical TV norm to higher dimensions, TGV allows recovering images with sharp edges and exempted from staircasing effect. Another approach to ameliorate this effect, an improved model constructed by the second-order bounded variational space $B V^{2}$, was proposed in [12], [13]. The use of this technique also substantially reduces the staircasing effect while preserving edges. In particular, the second-order space $B V^{2}$ shows convincing results in image denoising [12], and in image texture extraction [13].

The second-order variational model of Rudin-Osher-Fatemi (ROF2) was given as follows:

$$
\inf _{u \in B V^{2}(\Omega)} \frac{1}{2 \lambda}\|f-u\|_{L^{2}(\Omega)}^{2}+T V^{2}(u, \Omega)
$$


where the $T V^{2}(u, \Omega)$ represents the second-order total variation defined by:

$$
T V^{2}(u, \Omega)=\sup _{\phi}\left\{\langle\nabla u, \operatorname{div} \phi\rangle_{\mathbb{R}^{n}}: \phi \in C_{c}^{2}\left(\Omega, \mathbb{R}^{n \times n}\right),\|\phi\|_{\infty} \leq 1\right\},
$$

and the space of second-order bounded variation $B V^{2}(\Omega)$ is then defined as the space of all functions $u \in W^{1,1}(\Omega)$ such that $T V^{2}(u, \Omega)<+\infty$, endowed with the norm:

$$
\|u\|_{B V^{2}(\Omega)}=\|u\|_{W^{1,1}(\Omega)}+T V^{2}(u, \Omega) .
$$

This functional space is first introduced in [12], [15]. We also notice that problem (4) has a unique solution by the same lower semi-continous and strictly convex properties of the energy as $B V^{2}$.

The image decomposition with ROF2 is aims at dividing an image $f$ into two components $u$ and $v$, which represent the piecewise-smooth and oscillatory component, respectively. Let us consider the discrete decomposition model is described by minimizing a convex functional which depends on the two variables $u$ and $v$ as:

$$
\inf _{(u, v) \in X \times X} \frac{1}{2 \lambda}\|f-u-v\|_{X}^{2}+J_{2}(u)
$$

for a certain tuning parameter $\lambda>0$ and $J_{2}$ stands for the discretization of $T V^{2}$.

This problem is well mentioned in the past work of Tran et al. in [16], in which the model of ROF2 and wavelet shrinkage are combined together to separate an image into two parts: the cartoon component $u$ and the remaining oscillatory component $v$. In addition to $u+v$ (structure plus oscillation) decompositions, one can also consider introducing a noise component $\omega$, which leads to a $u+v+\omega$ decomposition consisting of structure, texture and noise respectively. Recently, such a decomposition was introduced by Aujol J.F. and Chambolle A. in [10], where the use of ROF model and wavelet shrinkage are well adopted. The idea here is to extend the $B V$-norm in ROF model to the $B V^{2}$-norm in ROF2 model, that better captures geometrical structures. In the main research of this paper, section 2, our new approach are clearly outperformed and applied in consideration of three components of decomposition algorithm. Furthermore, since the oscillatory components do not have small $L^{2}$ norms as noted in [18], the use of the $L^{2}$ norm is not a suitable choice for the variable containing the oscillations of the image. It was presented in [22], [14] that the weaker $G$-norm as the dual norm of $W^{1,1}$ that capture the pattern of oscillation component successfully. More precisely, in our approach, the smooth component is considered in the space of second-order bounded variation $B V^{2}$, and the textures or oscillating patterns are considered in $G$-norm. Moreover, in this paper, we propose the model that further separate the texture from noise in the oscillating part based on the process on wavelet coefficients of a given image $f$. In particular, the use of non-decimated wavelet ("à trous" algorithm, refer to [17]) and decimated wavelet (Mallat's algorithm, refer to [19]) soft-thresholding (shrinkage) have been applied.

The rest of this paper is organized as follows. In the next section 2, we describe the decomposition model, in which two adaptive models are proposed in our image decomposition and corresponding algorithms are then presented. Section 3 gives a part of parameter selection 
in our proposed model. Section 4 will present some examples, and numerical experiments are given to illustrate the effectiveness of our approach. At last, some comments and concluding remarks are sketched out in section 5 .

\section{Image Decomposition model}

In the same spirit as in the image decomposition model introduced in [16], [12], by introducing one more part, the noise component $\omega$, we here also propose another decomposition model which consists of cartoon $u$, texture $v$ and the noise $\omega$. One follows the decomposition model in [10], that instead of the total variation, the second-order total variation regularization problem is considered. In the main objective of this paper, we consider decomposition model as minimizing the following functional energy:

$$
\min _{(u, v, w) \in X \times X \times X}\left\{\frac{1}{2 \lambda}\|f-u-v-\omega\|_{X}^{2}+J_{2}^{*}\left(\frac{v}{\mu}\right)+B^{*}\left(\frac{\omega}{\delta}\right)+J_{2}(u)\right\},
$$

where $J_{2}(u)$ represents the discretization of $T V^{2}(u, \Omega)$ as in [12], and $J_{2}^{*}$ is the LegendreFenchel transform of $J_{2}(\mathrm{read}[1])$, this is the indicator function of a closed convex set $K$ as:

$$
J_{2}^{*}(v)=\chi_{K}(v)= \begin{cases}0, & \text { if } v \in K \\ +\infty, & \text { otherwise }\end{cases}
$$

As in [14], the operator $B^{*}$ in (8) is defined by:

$$
B^{*}\left(\frac{\omega}{\delta}\right)= \begin{cases}0, & \text { if } \frac{\omega}{\delta} \in B_{E} \\ +\infty, & \text { otherwise }\end{cases}
$$

where $B_{E}=\left\{v /\|v\|_{E} \leq 1\right\}$ and $E=\dot{B}_{-1, \infty}^{\infty}$ is the dual space of the standard Besov space $\dot{B}_{1,1}^{1}$, we cite here [10] for detail information.

The idea here is that the term $B^{*}\left(\frac{\omega}{\delta}\right)$ representing the $E$ norm. This term is used to model the noise, and it has been proved that the approximation is well suited to denoise the textured images for its tendency to preserve texture components, leads to [10], [21], [22].

Furthermore, as proved in [12], [11], the problem (8) admits a solution $\left(u^{*}, v^{*}, \omega^{*}\right)$, where $u^{*}$ and $v^{*}+\omega^{*}$ are unique. The proposed model (8) is then solved for cartoon + texture + noise by transforming it into the following three sub-problems as:

1. Subproblem 1: For $v$ and $\omega$ being fixed, we find $u$ as solution to:

$$
\min _{u \in X}\left\{J_{2}(u)+\frac{1}{2 \lambda}\|f-u-v-\omega\|_{X}^{2}\right\} .
$$

2. Subproblem 2: For $u$ and $\omega$ being fixed, we find $v$ as solution to:

$$
\min _{v \in \mu K}\|f-u-v-\omega\|_{X}^{2} .
$$


3. Subproblem 3: For $u$ and $v$ being fixed, we find $\omega$ as solution to:

$$
\min _{\omega \in \delta B_{E}}\|f-u-v-\omega\|_{X}^{2} .
$$

In the following subsections, one tries to obtain the discrete solution to each subproblem $\left(P_{1}\right)$, $\left(P_{2}\right)$ and $\left(P_{3}\right)$, respectively.

\subsection{Algorithm to solve the subproblem $\left(P_{1}\right)$}

In fact, the problem $\left(P_{1}\right)$ is the ROF2 model, this can be checked out easily in [12], [15]. In these references, it was proposed a nonlinear projection algorithm to minimize the ROF2 model, which is rewritten in the form of:

$$
\min _{u \in X}\left\{J_{2}(u)+\frac{1}{2 \lambda}\|f-u\|_{X}^{2}\right\}
$$

where $f$ is an observed image. For the finite dimensional optimization model, the following proposition holds.

Proposition 2.1. [15], [12] The solution to problem (11) verifies:

$$
u=f-P_{\lambda K}(f) ;
$$

where $P_{\lambda K}$ be the orthogonal projector operator on $\lambda K$, and

$$
K:=\left\{H^{*} p \mid p \in X^{N \times N},\left\|p_{i_{1}, i_{2}, \ldots, i_{n}}\right\|_{\mathbb{R}^{N \times N}} \leq 1 ; 1 \leq i_{1}, i_{2}, \ldots, i_{d} \leq N\right\} .
$$

One notices that in proposition 2.1, $H$ presents the Hessian operator and $H^{*}$ gives its adjoint. Moreover, in order to approximate the projection term $P_{\lambda K}(f)$, the following problem has to be considered, [15]:

$$
\left\{\begin{array}{l}
\min _{p \in X^{N \times N}}\left\{\left\|\lambda H^{*} p-f\right\|_{X}^{2}\right\}, \\
\left\|p_{i_{1}, i_{2}, \ldots, i_{d}}\right\|_{\mathbb{R}^{N \times N}}^{2} \leq 1 ; \quad 1 \leq i_{1}, i_{2}, \ldots, i_{d} \leq N .
\end{array}\right.
$$

The discrete problem (14) can be solved by the fixed point method with an iterative scheme on the solution $p$ satisfies: $p^{0}=0$ and

$$
p_{i_{1}, i_{2}, \ldots, i_{d}}^{n+1}=\frac{p_{i_{1}, i_{2}, \ldots, i_{d}}^{n}-\tau\left(H\left[H^{*} p^{n}-\frac{f}{\lambda}\right]\right)_{i_{1}, i_{2}, \ldots, i_{d}}}{1+\tau\left\|\left(H\left[H^{*} p^{n}-\frac{f}{\lambda}\right]\right)_{i_{1}, i_{2}, \ldots, i_{d}}\right\|_{\mathbb{R}^{N \times N}}}, \quad n=0,1,2,3, \ldots
$$

The discretization of the Hessian operator $H$ and its adjoint operator $H^{*}$, as well as a sufficient condition ensuring the convergence of the algorithm can be found in $[12,13]$. It is shown that in two dimensions, as long as $\tau \leq 1 / 64$, the term $\lambda\left(H^{*} p^{n}\right)_{n}$ converges to $P_{\lambda K}(f)$ as $n \rightarrow \infty$.

As a consequence, the approximate solution to $\left(P_{1}\right)$ is given by:

$$
u^{*}=f-v-\omega-P_{\lambda K}(f-v-\omega) .
$$




\subsection{Algorithm to solve the subproblem $\left(P_{2}\right)$}

This section dedicates to solve the discrete problem $\left(P_{2}\right)$ as a projection on the closed convex set $K$. Refer to [1, 10,22], the approximate solution can be found in terms of:

$$
v^{*}=P_{\mu K}(f-u-\omega) .
$$

where $P_{\mu K}$ denotes the orthogonal projector operator on the set $\mu K$, the given $K$ in (13).

As already mentioned in the section 2.1, one approximates the projection $P_{\mu K}(f)$ as the solution of problem (14) by the fixed point algorithm as in (15). Finally, the discrete solution of $\left(P_{2}\right)$ are fixed in (17).

\subsection{Algorithm to solve the subproblem $\left(P_{3}\right)$}

The approximate solution to sub-problem $\left(P_{3}\right)$ is given by:

$$
\omega^{*}=P_{\delta B_{E}}(f-u-v),
$$

where $\delta B_{E}=B_{E(\delta)}=\left\{\omega:\|\omega\|_{E} \leq \delta\right\}$. In order to approximate the projection $P_{\delta B_{E}}$, one considers the dual problem of $\left(P_{3}\right)$ with the solution $z$ to:

$$
\min _{z \in X}\left(\frac{1}{2}\|f-u-v-z\|_{X}^{2}+\delta\|z\|_{\dot{B}_{-1,1}^{1}}\right)
$$

and the approximate solution $z^{*}$ is given from a wavelet soft/hard thresholding algorithm with threshold $2 \delta$. It can be rewritten as $z^{*}:=W S T(f-u-v, 2 \delta)$. Furthermore, in numerical experiments, one applies the decimated and non-decimated wavelet shrinkage to obtain comparable results. Therein,the Haar wavelet (haar), Daubechies wavelet with eight vanishing moments $(d b 8)$ and the à trous wavelet are applied,respectively with a selected threshold parameter $2 \delta$ is chosen referring to [5].

The approximate solution $\omega^{*}$ to sub-problem $\left(P_{3}\right)$ is then given by:

$$
\omega^{*}=f-u-v-W S T(f-u-v, 2 \delta) .
$$

\subsection{Algorithm for decomposition model}

We finally propose an iterative algorithm for image decomposition $u+v+w$ model as follows. 


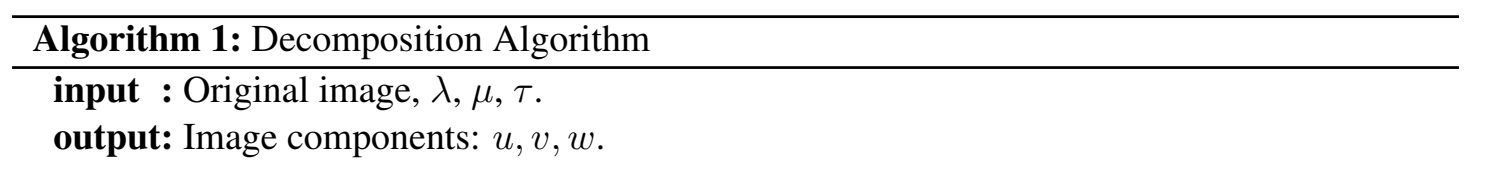

1. Initialization:

$$
u_{0}=v_{0}=\omega_{0}=0,
$$

2. Iterations on $n$ : update the $(n+1)^{t h}$ iterative solution from $\left(u_{n}, v_{n}, \omega_{n}\right)$ :

$$
\begin{aligned}
u_{n+1} & =f-v_{n}-\omega_{n}-P_{\lambda K}\left(f-v_{n}-\omega_{n}\right), \\
v_{n+1} & =P_{\mu K}\left(f-u_{n+1}-\omega_{n}\right), \\
\omega_{n+1} & =f-u_{n+1}-v_{n+1}-W S T\left(f-u_{n+1}-v_{n+1}, 2 \delta\right) .
\end{aligned}
$$

3. Stopping criterion:

$$
\max \left\{\left\|u_{n+1}-u_{n}\right\|,\left\|v_{n+1}-v_{n}\right\|,\left\|\omega_{n+1}-\omega_{n}\right\|\right\} \leq \varepsilon,
$$

where $\varepsilon$ represents a small positive number defined by users in numerical tests.

\section{Parameter selection}

According to the decomposition model (8) and its algorithm, it introduces additional parameters $\lambda, \mu$ and $\delta$ that control how the solution $(u, v, \omega)$ behaves. The choice of the fitting regularization parameters $\lambda, \mu$ in $\left(P_{1}\right)$ and $\left(P_{2}\right)$ in image restoration is still considered an important open problem. This was also discussed in the work of Bergounioux et al. in [12], [13].

Firstly, let us notice to the parameter $\lambda$ in the submodel $\left(P_{1}\right)$. The parameter $\lambda>0$ stands for regularization parameter, which controls the trade-off between a good fit of solution and a smoothness requirement due to the ROF2 model (11). In particular, it controls the $L^{2}$ norm of residual $f-u-v-\omega$. If $\lambda$ is small, the small residual norm we have. It can be found that in practice, this parameter is difficult to tune, it is chosen dependently upon the input image and additional the noise standard deviation $\sigma_{\text {noise }}$. Tuning $\lambda$ often relies on experience and visual inspection. We do not have any automatic way for choosing $\lambda$ as far as we know. In some numerical examples as below, one gives different values of $\lambda$ testing to several images. This is a very significant issue. In our experiments, we found that $0.5 \leq \lambda \leq 50$ is appropriate for gray scale images with intensities from 0 to 255 .

The parameter $\mu$ in sub-model $\left(P_{2}\right)$ represents the $L^{2}$-norm of the texture component $v$. This parameter controls the magnitude of $L^{2}$ norm, similar to the parameter $\lambda$ problem (14). The amount of noise removed from a given noisy image is controlled by these parameters. One leads to $[1,12]$ for some effectiveness choices of $\mu$ that make our algorithm be adapted well. The larger $\mu$ is, the more the information is averaged in the structural component $u+\omega$, and the more texture is extracted. However, the choice of $\mu$ depends upon the texture properties of the input image. 
In the sub-model $\left(P_{3}\right)$, we mention to the choice of threshold parameter in wavelet shrinkage. In (19), $\delta$ controls the $E$ norm of the $\omega$, amount of noise component. In image denoising, it has some proposed criteria for thresholding parameter selection. Some threshold selection methods are introduced for white Gaussian noise distributions with known variances. There are a lot of methods, such as SURE, Minimax methods ..., which can be found in [9], [8] and references therein. For instance, in 2D, the numerical calculation optimal threshold parameter for softthresholding is found by the popular universal method proposes the $T$ as follows:

$$
T=\sigma_{\text {noise }} \sqrt{2 \log \left(N^{2}\right)}
$$

where $N^{2}$ is the number of data points and $\sigma_{\text {noise }}^{2}$ stands for the variance of Gaussian white noise. Even if this threshold parameter is good, it does not handle for every type of image. In fact, for images with sharp edges, the use of this shrinkage parameter often leads to an over-smoothed geometrical component. For the better visualization, we adopt the approach chosen by Aujol and Chambolle in [10], parameter $\delta$ in (20) is often chosen as

$$
\delta=\frac{T}{2}=\frac{\sigma_{\text {noise }} \sqrt{2 \log \left(N^{2}\right)}}{2} .
$$

Or to get the even better results, one defines a weighting parameter $\operatorname{\eta in}(0,1]$ and set

$$
\delta=\eta \frac{\sigma_{\text {noise }} \sqrt{2 \log \left(N^{2}\right)}}{2} .
$$

As a consequence, the choices for these parameters follow the same guidelines as above together with numerical approximations. These parameters depend on the input clean or noisy images. One points out how experimental results are performed in the next section.

\section{Numerical experiments}

In this section, many types of images are considered: images with textured structures, images with complicated details. Let us divide this numerical experiment into two competitive applied algorithms, where the two wavelet shrinkages are applied: à trous algorithm and Mallat's algorithm. The grayscale images of size $512 \times 512$ and $256 \times 256$ are used for image decomposition experiments: "barbara, baboon, gold-hill" and the square cut-off part of image barbara. The last image is formed by texture elements, that called "texture+structure" image. The original images are shown in figure 1 . 


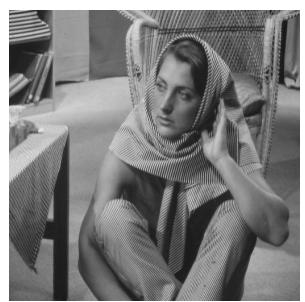

(a) Barbara, $512 \times 512$.

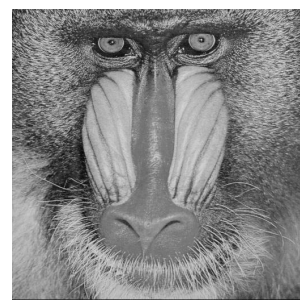

(b) Baboon, $512 \times 512$.

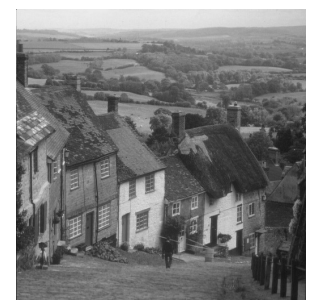

(c) Gold-hill, $512 \times$ 512 .

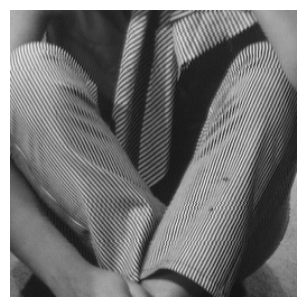

(d) Cut-off image of Barbara, $256 \times 256$.

Figure 1: Original images.

\subsection{Experiment 1: Input no-noise images}

In order to test the algorithm 2.4, we first apply to the no-noise images. It points out in figure 2 below some numerical results that applying the wavelet "à trous" algorithm to the problem $\left(P_{3}\right)$. The final scale is chosen as 4 in the wavelet orthogonal decomposition.

We present some numerical experiments for a visual comparison of results for test images. Figure 3 shows results are well performed with Mallat's wavelet algorithm. Wavelet transform employs Haar and Daubechies wavelet with eight vanishing moments $(\mathrm{db} 8)$, with four scales of orthogonal decomposition. In addition, the iteration termination condition is chosen with $\varepsilon=10^{-4}$ in our algorithm for the input large size image. Note that in results presented below, one adds a constant to $v$ and $\omega$ for better illustration purpose.

It can be seen that the component $u$ by our proposed model exhibits almost no artifacts, even around the edges, thus providing a very high visual quality. As in [1] and [10], it is also well known that the classical regularization ROF model producing staircase-like artifacts, the cartoon component has undesirable piecewise constant regions. One may have a look on some numerical experiments given therein to get compatible conclusion. The appearance of ROF2 model in our decomposition algorithm substantially reduces this effect. It is also important to point out that the absence of visual artifacts is proved in all the performed experiments. In this paper, authors do not give image decomposition results with ROF model, whereas one has known results in [10]. In addition, the advantages of ROF 2 model compared with ROF model is well proved in [12], [13] and authors presented several numerical experiments therein.

Results distinctly illuminate that the images decomposed by our advanced algorithm in texture component $v$ are better than those of applied total variation regularization scheme. For the cut-off part of barbara image, one considers a real texture image without noise, the residual component is much less geometry and texture than by the applied ROF scheme. This model separates the texture details shown in $v$ from non-textured images kept in $u$, one sees that the wrinkles of barbara's clothes are shown in the component $v$. Moreover, one can say that results of $w$ by Mallat's wavelet algorithm is much better than the "à trous" wavelet. In figure 2 and 3, it can be seen in third column that the component $w$, supposed to be the oscillating part, have been shown as almost zeros images for Mallat's wavelet transform scheme. 


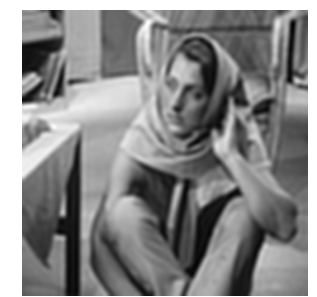

(a) $u$.

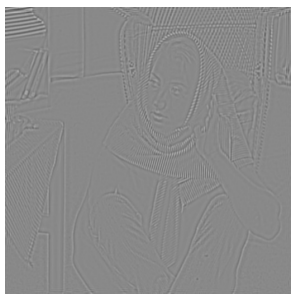

(d) $v$.

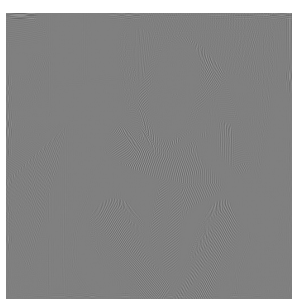

(g) $w$.

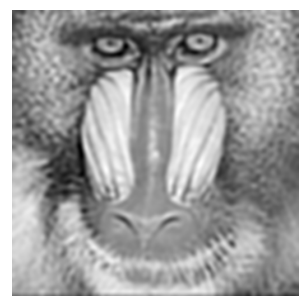

(b) $u$.

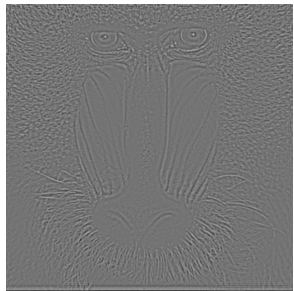

(e) $v$.

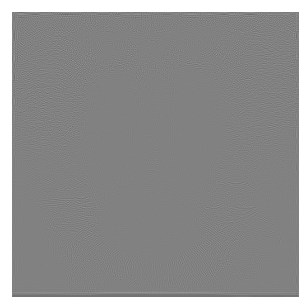

(h) $w$.

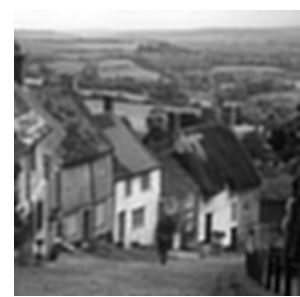

(c) $u$.

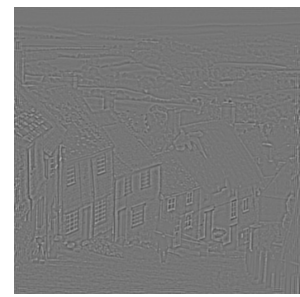

(f) $v$.

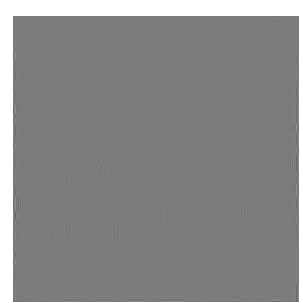

(i) $w$.

Figure 2: Example with Barbara, Baboon and the Goldhill images, $\lambda=10, \mu=50$, using the "à trous" wavelet shrinkage.

\subsection{Experiment 2: Input noisy images}

In this section, one considers the performance of decomposition model to input noisy images. With the additive noise, the input image is assumed to be the summation of original (clean) image and an additive random noise. It also notices that the random noise has Gaussian distribution with a fixed standard variance $\sigma_{\text {noise }}^{2}$.

We repeat the iterative decomposition algorithm to the input noisy image $f$, and the value of $\eta$ here is chosen as 0.1. By the theory of model, it assumes that input image is decomposed into three components $u, v$ and the $\omega$, where the $\omega$ keeps the estimated noise. As a result, the distribution of estimated noise should approach that of the additive noise.

One gives some numerical results in figures 5 and 6 for different value of $\sigma$. It also remarks here that we only perform numerical solutions for the cut-off Barbara, as in figure 3(a). One chooses the Barbara as its nice mixture of details, flat regions, shading areas, and textural features. Results are checked out for a chosen noise power estimated with standard deviation $\sigma=5$ and $\sigma=20$, respectively. Similar to the previous section 4.1, the iteration termination condition 


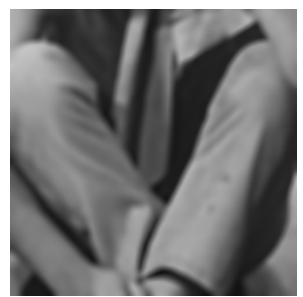

(a) Haar, $u$.

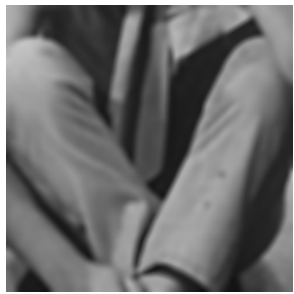

(d) Daubechies-8, $u$.

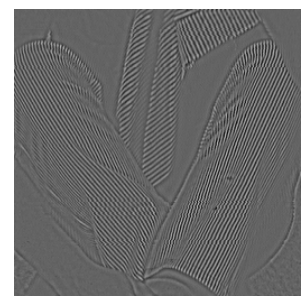

(b) Haar, $v+100$.

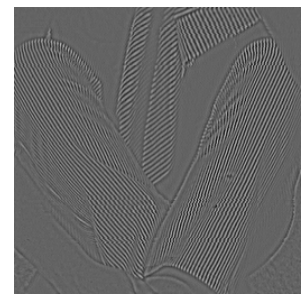

(e) Daubechies- $8, v+$ 100 .

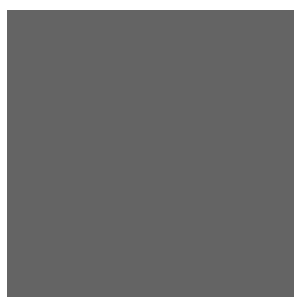

(c) Haar, $w+100$.

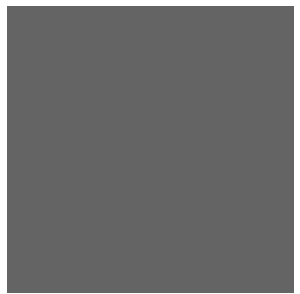

(f) Daubechies-8, $w+$ 100 .

Figure 3: Example with a part of Barbara, $\lambda=10, \mu=50$, using the Mallat's wavelet shrinkage.

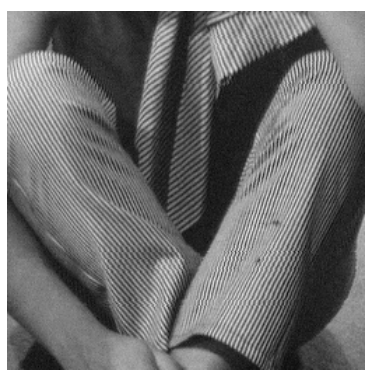

(a) $\sigma=5$.

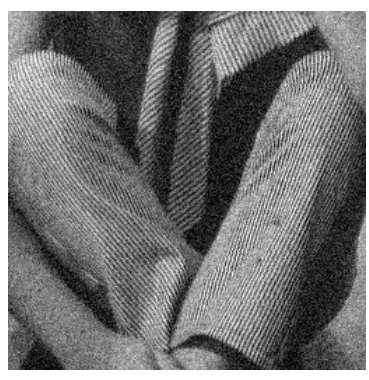

(b) $\sigma=20$.

Figure 4: Noisy image for $\sigma=5,20$.

for decomposition problem is implemented by choosing $\varepsilon=10^{-4}$ for the input large size and noisy images.

Figures 5 and 6 (b) to (g) depict the decomposition results given by our approach with $\sigma=5$ and $\sigma=20$, respectively. Results are displayed in three columns respected to cartoon component $u$, the textural component $v$ and the residual component $w$. For better visualization, the $v$ and $w$ component are displayed as $v+100$ and $w+100$. Consider smooth components $u$ in the first columns, texture and noise have been successfully removed in $u$. More texture appears in the $v$ component by the proposed model.

It can be seen that our model removed noise together with texture from input noisy image $f$. Our estimations retain good visual quality. In addition, the cartoon part $u$ in these results give no stair-case effect and correct edge locations. Observing in the figures 5 and 6 , for low level of noise, there are very few disturbing artifacts in component $u$, and one has the good preservation 
of sharp details (legs of barbara), regular structures (pants of barbara) in the component $v$. This aims that the decomposition model applied second variational model ROF2 makes difference with classical model with ROF, [10].

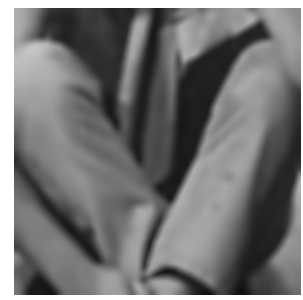

(a) "À trous", $u$.

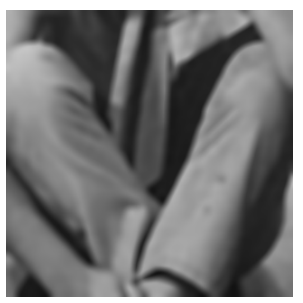

(d) Haar, $u$.

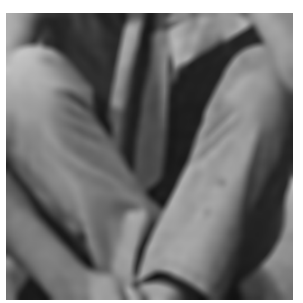

(g) Daubechies-8, $u$.

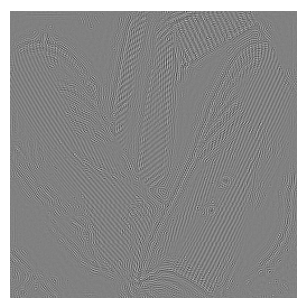

(b) “À trous", $v+100$.

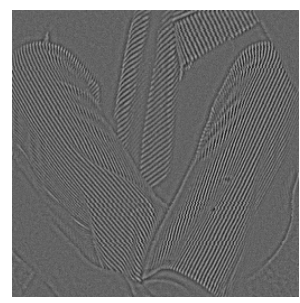

(e) Haar, $v+100$.

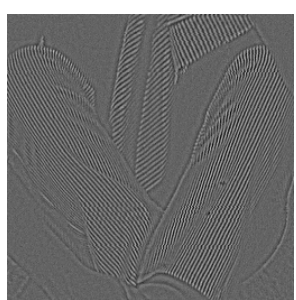

(h) Daubechies-8, $v+$ 100.

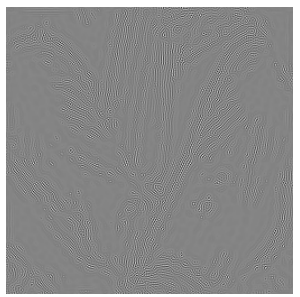

(c) “À trous”, $w+100$.

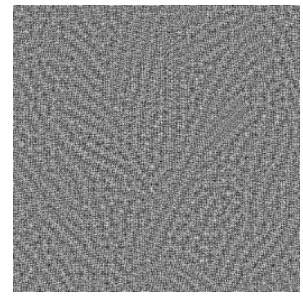

(f) Haar, $w+100$.

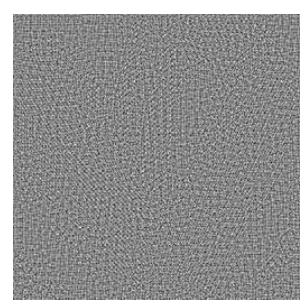

(i) Daubechies-8, w+ 100 .

Figure 5: Image decomposition with noisy image, for $\sigma=5, \lambda=10, \mu=50$.

In Figure 7, we present a computational cost for one of the previous examples. We measure the error between two consecutive iterations, as the norm $\frac{\left\|u_{n+1}-u_{n}\right\|}{\left\|u_{n}\right\|}$ at each iterative step $n$, and plot them with the iterative computational time. The error changes substantially between consecutive iterations. Here, the computational time is represented as a function of this error. 


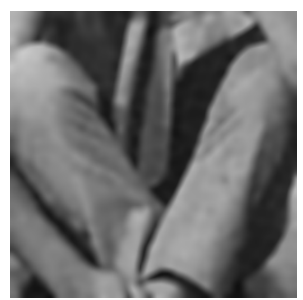

(a) Haar, $u$.

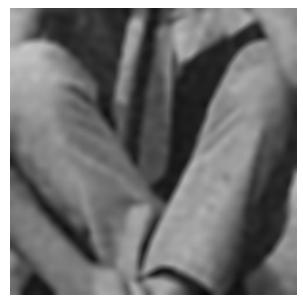

(d) Daubechies-8, $u$.

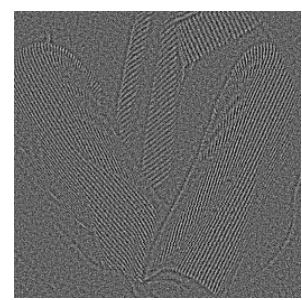

(b) Haar, $v+100$.

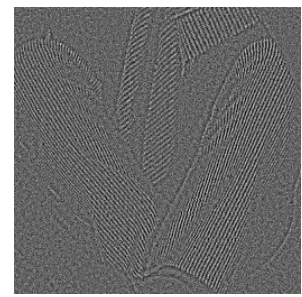

(e) Daubechies- $8, v+$ 100.

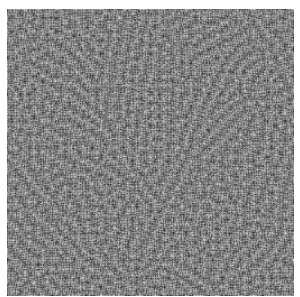

(c) Haar, $w+100$.

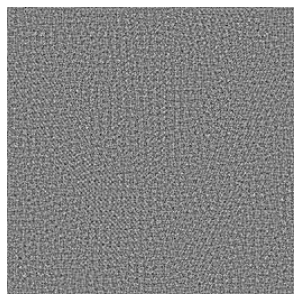

(f) Daubechies-8, $w+$ 100 .

Figure 6: Noisy image with $\sigma=20, \lambda=10, \mu=50$, applying (haar and $d b 8$ ) wavelets.

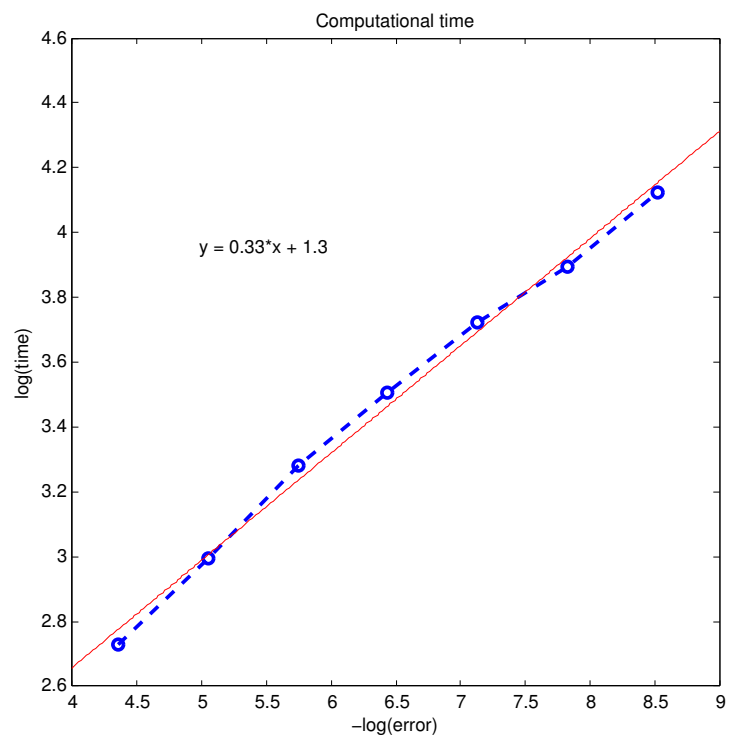

Figure 7: Computational cost.

\section{Conclusion}

Decomposing image into meaningful components is a challenging inverse problem in image processing. In this paper, as an original extension of the three components image decomposition algorithm of Aujol et al. in [10], we have introduced a new image decomposition model which 
incorporates the advantages of two preceding models: the ROF2 model and wavelet shrinkages. The proposed regularization model exhibits good performance in restoring the observed noisy image free from staircasing effect. In addition, wavelet shrinkage is used to separate the oscillating part due to texture from that due to noise. Two wavelet transforms, the decimated and non-decimated are considered separately in this work to obtain comparable results. The experimental results are quite promising. From the checked numerical experiments, one can realize that this model yields a convincing and appealing results. However, further work will focus on the estimation of the optimal algorithmic parameters for better texture and cartoon recovery. Moreover, some other interesting works are done in [3], [4], where different functional spaces are considered and may be alternative to the $B V^{2}$ space. Accompanies the use of second-order total variation $T V^{2}$ to overcome the limitation of $T V$, the Total Generalized Variation (TGV) was recognized as a well established higher-order generalization. It is an interesting issue to test the performance of the second-order TGV, for comparing the different decomposition algorithms. These further works will be described in a forthcoming paper.

\section{References}

[1] Chambolle A. An algorithm for total variation minimization and applications. JMIV, 20:89-97, 2004.

[2] Haddad A. and Meyer Y. An improvement of rudin-osher-fatemi model. Appl. Comput. Harmon. Anal., 22(3):319-334, 2007.

[3] K. Bredies. Recovering piecewise smooth multichannel images by minimization of convex functionals with total generalized variation penalty. Lecture Notes in Computer Science, 8293:44-77, 2014.

[4] Kristian Bredies, Karl Kunisch, and Thomas Pock. Total generalized variation. SIAM J. Img. Sci., 3(3):492-526, September 2010.

[5] A. Chambolle, R.A. De Vore, N. Lee, and B.J. Lucier. Nonlinear wavelet image processing: variational problems, compression, and noise removal through wavelet shrinkage. IEEE Trnascations on Image Processing, 7(3):319-355, 1998.

[6] Tony F. Chan, Antonio Marquina, and Pep Mulet. High-order total variation-based image restoration. SIAM J. Scientific Computing, 22(2):503-516, 2000.

[7] Ambrosio L.; Fusco N.; Pallara D. Functions of bounded variation and free discontinuity problems. Oxford Mathematical Monographs. The Clarendon Press Oxford University Press, New York, 2000.

[8] D.L. Donoho. Denoising by soft thresholding. IEEE Trans. on Imformation Theory, 41:613-627, 1995.

[9] D.L. Donoho and I. Johnstone. Ideal spatial adaptation via wavelet shrinkage. Biomedia, 81:425-455, 1994. 
[10] Aujol J.F. and Chambolle A. Dual norms and image decomposition models. IJCV, 63(1):85-104, 2005.

[11] Bergounioux M. On poincaré - wirtinger inequalities in spaces of functions of bounded variation. preprint, hal-00515451, 10 June 2011.

[12] Bergounioux M. and Piffet L. A second-order model for image denoising. Set-Valued Analysis and Variational Analysis, 18(3-4):277-306, 2010.

[13] Bergounioux M. and Tran M.P. A second-order model for 3d texture extraction. Mathematical Image Processing, Springer Proc. Math., 5:41-57, 2010.

[14] Yves Meyer. Oscillating Patterns in Image Processing and Nonlinear Evolution Equations: The Fifteenth Dean Jacqueline B. Lewis Memorial Lectures. American Mathematical Society, Boston, MA, USA, 2001.

[15] Tran M.P. 3D image analysis with variational methods and wavelets - Applications to medical image processing. $\mathrm{PhD}$ thesis, $\mathrm{PhD}$ Thesis, École Doctorale Mathématiques, Informatique, Physique Théorique et Ingénierie des Systèmes, Laboratory MAPMO, University of orléans, 2012.

[16] Tran M.P., Péteri R., and Bergounioux M. Denoising 3d medical images using a second order variational model and wavelet shrinkage. Image Analysis and Recognition, Lecture Notes in Computer Science, 7325, Springer Berlin Heidenberg:138-145, 2012.

[17] Unaldi N. and Asari V.K. Undecimated wavelet transform-based image interpolation. ISVC 2010, (III):474-483, 1989.

[18] Leonid I. Rudin, Stanley Osher, and Emad Fatemi. Nonlinear total variation based noise removal algorithms. Phys. D, 60(1-4):259-268, November 1992.

[19] Mallat S. A theory for multiresolution signal decomposition: The wavelet representation. Computerized Medical Imaging and Graphics, 28:235-246, 2004.

[20] Otmar Scherzer. Denoising with higher order derivatives of bounded variation and an application to parameter estimation. Computing, 60(1):1-28, 1998.

[21] Chan T.F., Esedoglu S., and Parky F. Image decomposition combining staircasing reduction and texture extraction. Journal Vision and Communication Image, 18:464-486, 2007.

[22] Luminita A. Vese and Stanley J. Osher. Modeling textures with total variation minimization and oscillating pattern in image processing. Journal of mathematical Imaging and Vision, 20:7-18, 2004. 